\title{
MULTI-MODEL STABILITY CONTROL METHOD OF UNDERACTUATED BIPED ROBOTS BASED ON IMBALANCE DEGREES
}

\author{
Zhang, Z.-H. \& Hu, C. \\ Ningbo Institute of Technology, Zhejiang University, Ningbo, Zhejiang, China, 315100 \\ E-Mail: zzh6488@163.com
}

\begin{abstract}
In this paper, a stability control strategy for underactuated biped robots is proposed based on imbalance degree. The dynamic models of single-leg support of underactuated biped robots are firstly illustrated. Based on the external disturbance force strength of the system, the motion process of an underactuated biped robot is partitioned into three stages according to the imbalance degree. In different stages, corresponding dynamic models, tracking, gesture and gait switching control are adopted. Analysis of simulation result shows that the proposed underactuated gait control method is stable, practicable in engineering, and satisfies the real-time requirement.

(Received in November 2014, accepted in May 2015. This paper was with the authors 2 months for 1 revision.)
\end{abstract}

Key Words: Underactuated Biped Robots, Varying Gait, Imbalance Degree, Multi-Model Control

\section{INTRODUCTION}

Robots are born in the hope that it can think and behave like human beings. With the constant broadening of their application, robot technology has been beyond the scope of specific industrial robots. From the mid-1980s, driven by the computer technology, robot technology and artificial intelligence, research on intelligent robots have been carried out over the world. However, due to the lack of necessary knowledge on several aspects, such as the obtainment of machine intelligence, artificial intelligence theories, and information acquisition and processing, the research has fallen into a trough. Later, researchers realize that proposing a generic, general method of solving intelligence problems is unrealistic at present. Therefore, attention is paid on humanoid robots with specific functions [1].

Humanoid robot is a hotspot in robot research field [2, 3]. Humanoid robot prototypes with different function features have been constantly introduced in the world. However, in terms of applications at home and abroad, few have gone out of laboratories and come into real applications. Currently, Japan's Hongda and Toyata Corps. carried out the same level of research and development of humanoid robots. These robots serve as exemplary application in their Science and Technology Museums. Others are basically still at the laboratory prototype stage. However, this humanoid robot is fully actuated humanoid robots [4, 5].

Underactuated humanoid robots (UHRs) are mainly applied to aerospace, manufacturing production, etc. Since they have no drivers or motors, they are light weight, and have low power consumption, compared to actuated humanoid robots. Therefore, they have special significance in aerospace and manufacturing production. Study on them is very important to socio-economic development and technological innovation. Especially in the space or on the moon, reducing the number of motors and drivers is so important that it not only reduces the cost, but also saves energy and reduces the weight, significantly increasing the reliability.

According to the current research, foreign universities and institutes have made great efforts to establish humanoid robots. From the novelty search results, the leading international universities include UT, Carnegie Mellon University (CMU) and Delft University [6-10]. 


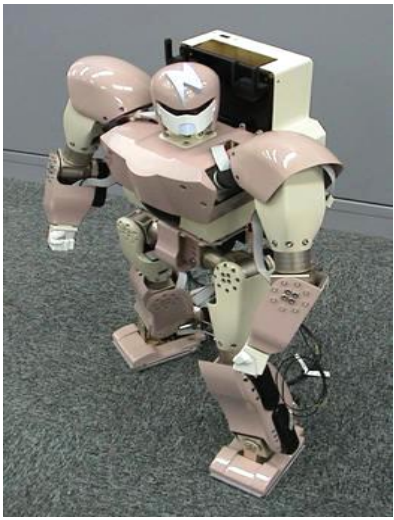

a) UHR prototype of UT;

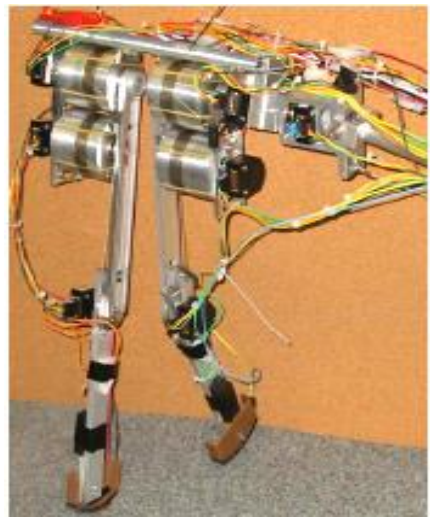

b) Biped robot prototype of CMU;

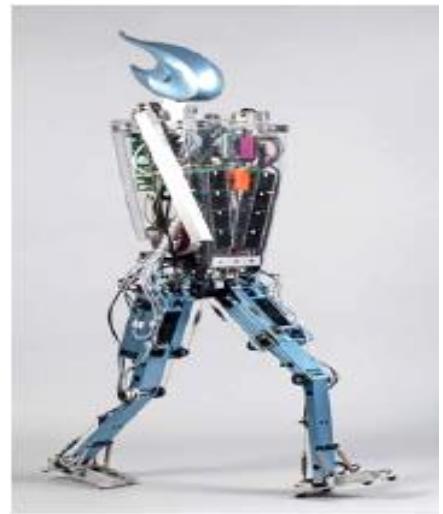

c) Robot Flame of Delft

Figure 1: Studied prototype of foreign universities.

Fig. 1 a shows the UHR prototype of UT, Fig. $1 \mathrm{~b}$ shows the biped robot prototype of CMU, and Fig. $1 \mathrm{c}$ shows the Robot Flame of Delft. Study on interference is based on relatively small interference, which is an ordinary method.

Some domestic research institutes are now carrying out studies on shape similar humanoid robots, and much work has been done. For example, Beijing Institute of Technology's patent application: Method and device of upper body posture control for stable walking of humanoid robots (China Patent Database CN101414189), which introduced a method and a device of upper body posture control for stable walking humanoid robots. The described method includes: Judge the current status of the robot's feet, which refers to the support state or swing state; Calculate the correct value of robot hip joints according to the feet status; Calculate the servo reference value of the hip joints according to the reference value. The described device includes judging module, the first calculation module, and the second calculation module. The described method adjusts the upper body posture by adjusting hip joints, in order to stabilize the walking robots. The method does not need specified mathematic robot models and it has a wide range of applications. It is easy in calculation, and has small delay compensation, keeping the dynamic stability of robots in real time [11].

Sheng and Wang of Department of Electrical and Mechanical Engineering and Automation, National Defense University, studied the erect posture stability control of point-contact biped walking robots. The authors proposed an erect posture stability control strategy for point-contact biped walking robots. They established a robot dynamic model and analysed the stability and controllability of the robot erect posture. They constructed a global fuzzy model of robots based on the Takagi-Sugeno (T-S) modelling, and designed a globally asymptotic stable fuzzy controller based on linear-quadratic regulator (LQR) optimal control theory to control the robot erect posture. Simulation results have shown that the algorithm has a wide stability range. It can make the robots rapidly restore upright posture after power-on or switching from walking to standing position, and keep the robots stable [12-15].

There have been many research results on the motion planning and control of actuated humanoid robots. However, there have been few studies on stability control of UHRs under strong interference.

The motion control and planning of UHRs is a difficult problem. There is no good motion control and planning method at present for UHRs, especially for strong and medium interference.

Since the weak interference for UHRs is very common, the control problems of UHRs mainly focus on this aspect. Under weak interference, the task of control is to minimize the error of each joint, and to make the UHRs' movements close to reference movements [16].

Actually, the stronger the interference is, the greater the danger of losing dynamic balance is. Therefore, the primary task is to prevent the UHRs from falling down. At this stage, UHRs 
are not directly involved in humanoid part. For example, the reference trajectories of arms being tracked should be abandoned and the dynamic balance should be maintained as much as possible, so that the trajectories of UHRs' legs do not dramatically change. In other words, some parts of UHRs abandon their internal cooperation to prevent falling down, in exchange for the possibility of continuing being humanoid. If the interference is strong enough to make UHRs generate underactuated joints and overturn UHRs, all joints should abandon their internal cooperation and attempt to maintain dynamic balance [17].

The contact area of the legs of UHRs and the ground is very likely to degenerate into lines or points, so that sensors cannot detect the contact force. In this situation, ZMP point does not exist. To determine how to compensate or re-establish internal cooperation, information of other sensors are needed, especially information about the relative positions of UHRs. For example, gyroscopes, accelerometers, tilt sensors, etc. In short, temporary compensation actions planned by other information are needed. Therefore, the control task of humanoid robots is to take any measures, if possible, to prevent robots from falling down; then adjust them to reduce the difference between actual movement and reference movement, making humanoid robots gradually return to the reference movement and maintain dynamic balance.

Focusing on strong and medium interference, a multi-model stability control method based on imbalance degrees is proposed to maintain the dynamic balance of UHRs.

\section{MULTI-MODEL CONTROL METHOD}

Lower extremity of the biped robot using standard configuration with 5 degrees of freedom, in this paper, we mainly consider forward walking control, walking in the process of always maintain hip joint rotation fixed position, so modelling ignore rotation of the hip joint, biped robot on lower limb joint structure model as shown in Fig. 2.

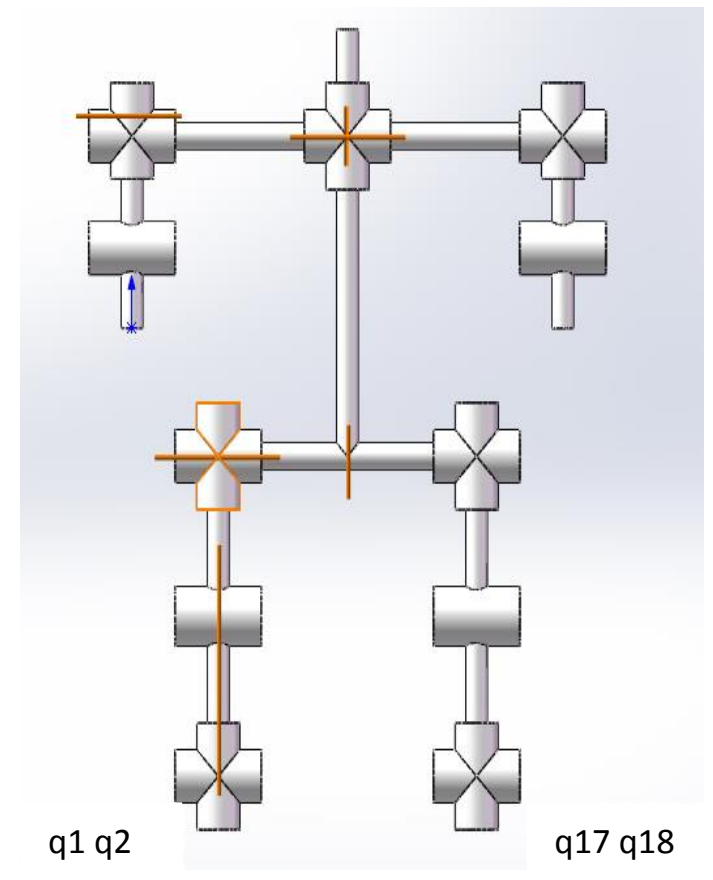

Figure 2: Schematic diagram of biped robot joint structure.

Biped humanoid robots use underactuated gaits; the walking process consists of a single-leg support stage and an instant double-leg support stage. The dynamic equation of single-leg stage in partitioned matrix form established based on Lagrange function is given by: 
where:

$$
\left[\begin{array}{lll}
M_{11} & M_{12} & M_{13} \\
M_{21} & M_{22} & M_{23} \\
M_{31} & M_{32} & M_{33}
\end{array}\right]\left[\begin{array}{l}
\ddot{q}_{A 1} \\
\ddot{q}_{G} \\
\ddot{q}_{A 2}
\end{array}\right]+\left[\begin{array}{l}
h_{1}(q, \dot{q}) \\
h_{2}(q, \dot{q}) \\
h_{3}(q, \dot{q})
\end{array}\right]=\left[\begin{array}{c}
\tau_{A 1} \\
\tau_{G} \\
\tau_{A 2}
\end{array}\right]
$$

$q_{A 1}=\left[\begin{array}{llll}q_{1} & q_{2} & \cdots & q_{5}\end{array}\right]^{T}$ are the joints of supporting leg. Let us suppose that $q_{1}, q_{2}$ are underactuated joint;

$\tau_{A 1}=\left[\begin{array}{llll}\tau_{1} & \tau_{2} & \cdots & \tau_{5}\end{array}\right]^{T}$ are the corresponding control moments;

$q_{A 2}=\left[\begin{array}{llll}q_{14} & q_{15} & \cdots & q_{18}\end{array}\right]^{T}$ are the joints of swinging leg. Let us suppose that $q_{17}, q_{18}$ are underactuated joint;

$\tau_{A 2}=\left[\begin{array}{llll}\tau_{14} & \tau_{15} & \cdots & \tau_{18}\end{array}\right]^{T}$ are the corresponding control moments;

$q_{G}=\left[\begin{array}{llll}q_{6} & q_{7} & \cdots & q_{13}\end{array}\right]^{T}$ are the joints of upper body;

$\tau_{G}=\left[\begin{array}{llll}\tau_{6} & \tau_{7} & \cdots & \tau_{13}\end{array}\right]^{T}$ are corresponding control moments;

$h=\left[h_{1}(q, \dot{q}) \quad h_{2}(q, \dot{q}) \quad h_{3}(q, \dot{q})\right]^{T}$ is the coupling term of Coriolis force, centrifugal force and gravity.

Taking $q=\left[q_{A 1} ; q_{G} ; q_{A 2}\right], x=[q ; \dot{q}]$, and $\tau=\left[\tau_{A 1} ; \tau_{G} \tau_{A 2}\right]$, the control model of biped robots is [2]:

$$
\dot{x}=\left[\begin{array}{c}
\dot{q} \\
-M(q)^{-1} H(q, \dot{q})
\end{array}\right]+\left[\begin{array}{c}
0 \\
M(q)^{-1}
\end{array}\right] \tau=f \overline{(x)}+g(x) \tau
$$

Fig. 3 shows the schematic of the proposed UHR control method. The CAN (Controller Area Network) bus can be used to implement the three kinds of controllers. Multi-model control is adopted for the cooperative control of different interferences (different imbalance degrees): C1 - leg angles controller, C2 - upper body posture angle controller, C3 - adjust gait controller, $x_{c o m}-$ mass centre position of the underactuated biped robot, $\dot{x}_{c o m}-$ mass centre velocity of the underactuated biped robot.

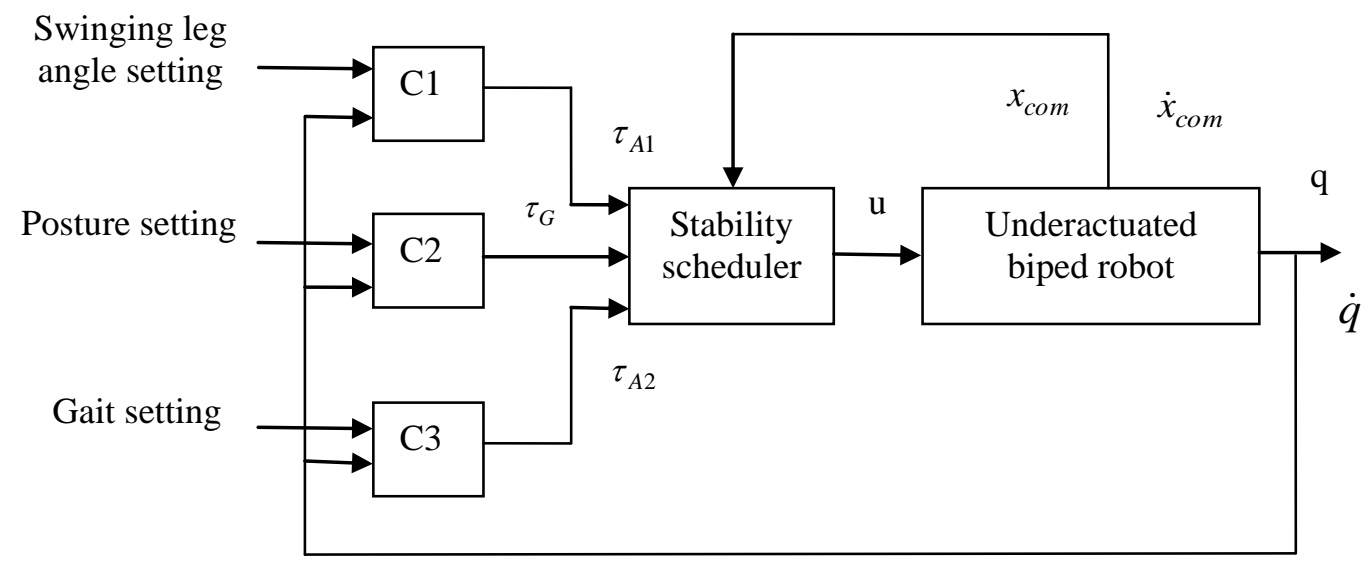

Figure 3: Stability control method for UHRs.

The controllers C1, C2, C3 are designed respectively according to different underactuated biped robot model1, model2, model3.The proposed technical scheme is the following steps of Underactuated Biped Robot walking pattern generation control method. The realization algorithm is provided.

Step 1: The establishment of underactuated biped robot motion control model. 


$$
\begin{gathered}
\dot{x}=f(x)+g(x) u \\
y=h_{0}(q)-h_{d}(\theta(q))=h(x)
\end{gathered}
$$

where $f(x), g(x)$ are nonlinear functions. $\theta(q)$ is system (attitude variable) synchronization variable. $h_{d}(\theta(q))$ is virtual constraint.

Step 2: Order constraint conditions as the system output.

$$
y=h_{0}(q)-h_{d}(\theta(q))=h(x)
$$

Step 3: The underactuated biped robot system is two order dynamic system using a feedback controller of underactuated biped robot control. The system of two differentials is as following:

$$
\frac{d^{2} y}{d t^{2}}=L_{f}^{2} H(q, \dot{q})+L_{f} L_{g} H(q) u
$$

where $H(q)$ is called the solution of the matrix, which is usually reversible.

Step 4: According to the feedback linearization control theory, the control input is:

$$
u(x)=\left(L_{g} L_{f} h(x)\right)^{-1}\left(v-L_{f}^{2} h(x)\right)
$$

Step 5: The nonlinear system is linearized into a dual integral system and the following expression is obtained:

$$
\ddot{y}=v
$$

Eq. (8) is the exact linearization of the dual integral system.

Step 6: Adopting finite time stability controller to control the underactuated biped robot system, we get the following expression:

$$
v=-k_{1} \operatorname{sign}(y)|y|^{\frac{a}{2-a}}-k_{2} \operatorname{sign}(\dot{y})|\dot{y}|^{a}
$$

where $k_{1}>0, k_{2}>0$ is convergence speed of the controller, and $0<a<1$ is the dynamic characteristics of the controller.

(1) When the interference is weak, i.e., the imbalance degree is small, $\mathrm{C} 1$ is used to adjust the leg angle to restore balance.

The gait controller adjusts the joint of swinging leg. It is the main part of realizing invariant gait control in underactuated state, or the main part of reference motion control; this situation is still within the safe supporting area. Appropriate control method can be designed to make the biped humanoid robots return to reference movements.

The design of $\mathrm{C} 1$ controller is mainly based on model design, i.e., based on underactuated multi-body dynamic model, with unconstrained control variables.

(2) When the interference is medium, i.e., the imbalance degrees are relatively big, $\mathrm{C} 2$ is used to adjust upper posture to restore balance.

The posture controller controls each joint of upper arm, and is the main part of realizing underactuated posture control; in this situation, interference causes the humanoid robots to deviate from the reference trajectories. To maintain dynamic balance, more effective measures should be taken for the biped humanoid robots. For example, control the swinging arms to make sure the robots are still in the safe supporting areas and let the robots gradually return to reference trajectories.

The design of C2 controller is mainly based on model design, but the control variables are constrained. Alternatively, it is based on modeless design. For example, adjusting a joint of upper arm or hip joints. 
(3) When interference is strong, i.e., the imbalance degrees are big, C3 is used to adjust steps to restore balance.

The Supporting leg joint angle controller controls each joint of the support leg, and it is the main part of realizing time-varying gait control for underactuated robots. In this situation, the attempt to reduce the deviation between joint trajectories and reference trajectories, and to make biped humanoid robots return to reference movements is meaningless, since the biped robots have lost their balance. Therefore, C3 is designed to prevent the biped robots from falling down. The design of $\mathrm{C} 3$ controller is mainly based on the fixed design of the original model.

\section{(4) Scheduling algorithm design}

The concept of imbalance degree is proposed. The imbalance degree $\rho$ is used to cooperatively adjust gaits and maintain stability. The imbalance degree $\rho$ reflects the strength of interference. Thresholds or fuzzy methods are used to divide the level of interference strength.

\section{IMBALANCE DEGREE AND MULTI-MODEL SCHEDULING}

Due to the complexity of the dynamic model of underactuated biped robots, we assume single supporting leg underactuated biped robots form linear inverted pendulum mode (LIPM) through virtual constraints.

The LIPM [2] system dynamic equation is:

$$
\ddot{x}=\frac{g}{z_{\text {com }}} x_{\text {com }}
$$

where $x_{\text {com }}, z_{\text {com }}$ are the horizontal position and height of the centroid, respectively; $g$ is the acceleration of gravity.

The imbalance degree of LIPM system is expressed as:

$$
\rho=\frac{1}{2} m^{2} g z_{\text {com }}\left(\operatorname{sign}\left(\dot{x}_{\text {com }}\right)\left(\dot{x}_{\text {com }}\right)^{2}+\frac{g}{z_{\text {com }}} \operatorname{sign}\left(x_{\text {com }}\right)\left(x_{\text {com }}\right)^{2}\right)
$$

where: $x_{\text {com }}$ - mass centre position of the underactuated biped robot, $\dot{x}_{\text {com }}-$ mass centre velocity of the underactuated biped robot. $z_{c o m}-$ mass centre high position of the underactuated biped robot.

If a robot in balance state is impacted by interference, the imbalance degree increases:

(1) When the interference is weak, the imbalance degree is low. If $|\rho|<\rho_{s \tan d 1}$, the balance can be restored by adjusting leg joint angles. Multi-model controller is C1, i.e. $u=\tau_{A 1}$.

(2) When the interference is relatively strong, the imbalance degree is relatively high. If $\rho_{s \tan d 1} \leq|\rho|<\rho_{s \tan d 2}$, the balance can be restored by adjusting the posture of upper body. Multi-model controller is $\mathrm{C} 2$, i.e. $u=\tau_{G}$.

(3) When the interference is very strong, the imbalance degree is very high. If $\rho_{s \tan d 2} \leq|\rho|<\rho_{\text {step }}$, the balance can be restored by adjusting steps. Multi-model controller is C3, i.e. $u=\tau_{A 2}$.

$\rho_{s \tan d 1}$ and $\rho_{s \tan d 2}$ are related to the stability control strategies of legs and postures, respectively. $\rho_{\text {step }}$ is related to the mechanical parameters and exercise capacity of robots.

The gait controller controls the joints of swinging legs, and is the main part of realizing the underactuated gait control. Time-varying gaits are adopted for controlling underactuated 
biped robots. The posture controller controls each joint of the arms of upper body, and is the main part of realizing the underactuated posture control. The angle controller for supporting legs controls each joint of supporting legs, and is the main part of realizing underactuated reference movement control.

The advantages of the proposed method with other reported in literature is multi-model controller based on the imbalance degree. A multi-model stability control method for underactuated biped robots is proposed based on imbalance degrees under strong and medium interference is considered. The main idea is to detect the position and speed of the centroid, and calculate the imbalance degrees. Corresponding control strategies are adopted according to the moving conditions of biped robots, in order to prevent falling down and restore posture and reference movement, eventually reaching a steady walking status.

Compare with the other three traditional approaches, and list the advantages and disadvantages in a Table I.

Table I: The advantages in comparing with the other three traditional approaches.

\begin{tabular}{|c|c|c|c|c|}
\hline$|\rho|<\rho_{s \tan d 1}$ & $\mathrm{C} 1$ & steady walking & fall & fall \\
\hline$\rho_{s \tan d 1} \leq|\rho|<\rho_{s \tan d 2}$ & $\mathrm{C} 2$ & fall & steady walking & fall \\
\hline$\rho_{s \tan d 2} \leq|\rho|<\rho_{\text {step }}$ & $\mathrm{C} 3$ & fall & fall & steady walking \\
\hline$|\rho|<\rho_{\text {step }}$ & Multi-model control & steady walking & steady walking & steady walking \\
\hline
\end{tabular}

\section{ANALYSIS AND DISCUSSION OF SCHEDULING SIMULATION}

Since the multi-model scheduler is the key of control, only the centroid position and speed of the biped robots are simulated in this study. The design of multi-model controller C1, C2, and $\mathrm{C} 3$ is presented in section 2 in the paper.

(1) Relation between imbalance degree and position and speed of centroid

The relation between LIPM system imbalance degree and the position and speed of the centroid is shown in Fig. 4.

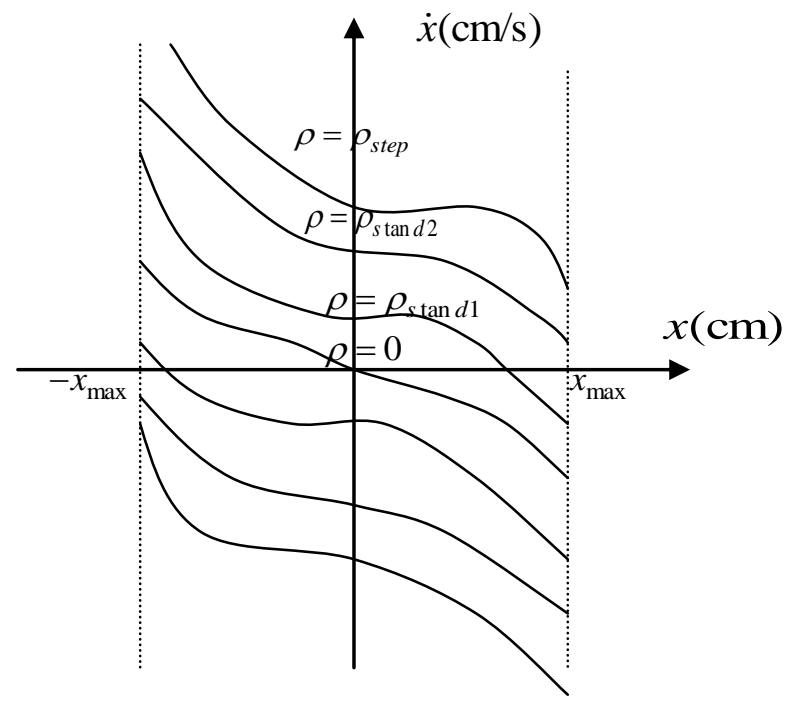

Figure 4: Relation between imbalance degree and position and speed of centroid.

It is clear that $\rho_{s \tan d 1}$ and $\rho_{s \tan d 2}$ are related to the stability control strategies of legs and postures, respectively. For example, when the position of centroid is relatively high, the allowed deviation distance is relatively big; when the moving speed of joints is relatively 
high, the leg swing time is relatively short, and the resistance of interference and impact is strong.

According to the decision on the stability of underactuated biped robots, the stability margin is defined as:

$$
\gamma=\rho_{\text {step }}-|\rho(x)|
$$

When $\gamma>0$, the robot is stable; when $\gamma \leq 0$, the current status is beyond system adjustment capacity, and the robot will fall down within the time of $\Delta t \quad(0 \leq \Delta t<\infty)$.

(2) Stability control simulation of underactuated biped robots

Assume the underactuated biped robot form the LIPM through virtual constraints [18, 19]. LIPM of the underactuated biped robot is shown in Fig. 5.

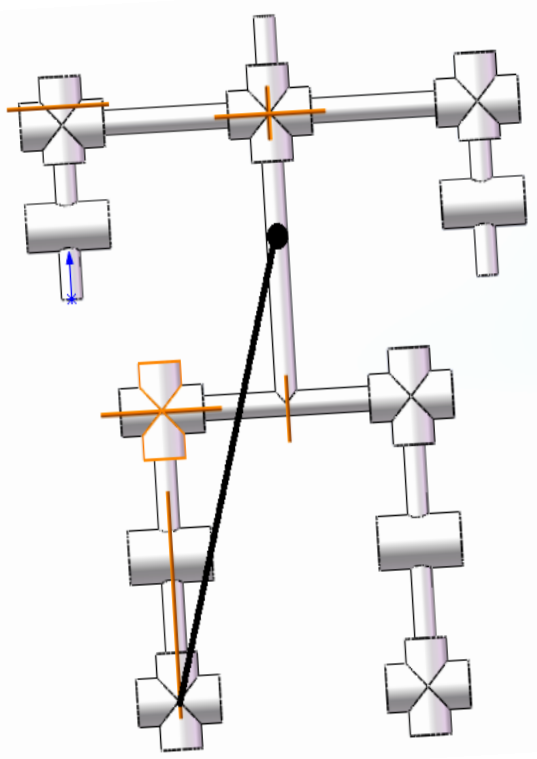

Figure 5: LIPM of the underactuated biped robot.

According to the differential equation describing the centroid horizontal movement:

$$
\ddot{x}=\frac{g}{z_{\text {com }}} x
$$

where $z_{\text {com }}$ is the horizontal position and height of the centroid. $g$ is the acceleration of gravity.

When $z_{\text {com }}$ is a constant, the following can be derived by solving the above differential equation:

$$
\begin{gathered}
x(t)=x(0) \cosh \left(t / T_{c}\right)+T_{c} \dot{x}(0) \sinh \left(t / T_{c}\right) \\
\dot{x}(t)=x(0) / T_{c} \sinh \left(t / T_{c}\right)+\dot{x}(0) \cosh \left(t / T_{c}\right)
\end{gathered}
$$

where $T_{c}$ is a constant depending on the height and gravity acceleration of the centroid. $x(0)$ and $\dot{x}(0)$ are the position and speed of the centroid at zero time, respectively, i.e., the initial conditions.

The underactuated biped robots flow chart of stability control method is shown in Fig. 6 .

Starting from the initial conditions, $x(0)$ and $\dot{x}(0)$, when the interference is relatively weak, the imbalance degree is relatively low. If $|\rho|<\rho_{s \tan d 1}$, the leg joint angle controller C1 can be used for adjustment. The adjusted position and speed of the centroid are $x(1)$ and $\dot{x}(1)$, respectively. 


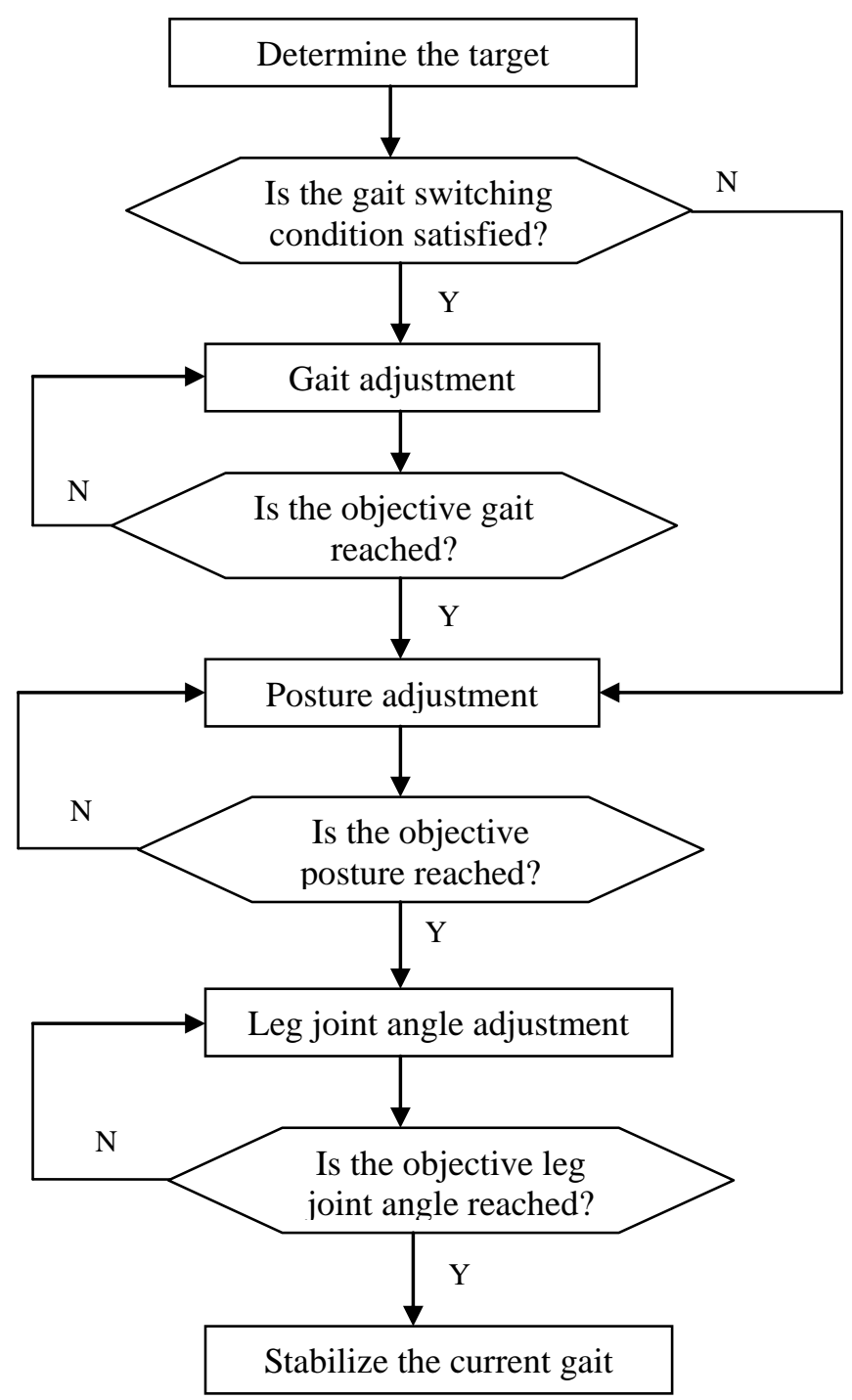

Figure 6: Flow chart of stability control method.

Starting from the initial conditions, $x(1)$ and $\dot{x}(1)$, when the interference is relatively strong, the imbalance degree is relatively high. If $\rho_{s \tan d 1} \leq|\rho|<\rho_{s \tan d 2}$, the upper body posture controller $\mathrm{C} 2$ can be used for adjustment. The adjusted position and speed of the centroid are $x(2)$ and $\dot{x}(2)$, respectively.

Starting from the initial conditions, $x(2)$ and $\dot{x}(2)$, when the interference is very strong, the imbalance degree is very high. If $\rho_{\text {stand } 2} \leq|\rho|<\rho_{\text {step }}$, the gait controller C3 can be used for adjustment. The adjusted position and speed of the centroid are $x(3)$ and $\dot{x}(3)$, respectively.

Finally, the responses of leg joint angle controller $\mathrm{C} 1$, upper body posture controller $\mathrm{C} 2$, and gait controller $\mathrm{C} 3$ constitute the total output response, which is shown in Fig. 7. The centroid position and speed are shown in Fig. 7 a and Fig. 7 b, respectively.

Table II is the initial states and running time of centroid position and speed in diffident stages.

It is clear in Figs. $7 \mathrm{a}$ and $7 \mathrm{~b}$ that with the initial condition $(-0.2,0.791)$, from $0 \mathrm{~s}$ to $0.8 \mathrm{~s}$, the leg joint angle controller $\mathrm{C} 1$ adjusts the centroid position and speed to $(-0.18,0.791)$. At $0.8 \mathrm{~s}$, there is a small step forward (switching legs). From $0.81 \mathrm{~s}$ to $1.6 \mathrm{~s}$, the upper body posture controller $\mathrm{C} 2$ adjusts the centroid position and speed to $(0.38,1.4)$. At $1.6 \mathrm{~s}$, there is a 
big step forward (switching legs). The initial condition is changed to $(-0.4,1.4)$. Then steps are continued. The centroid speed gradually decreases, and the centroid position gradually tends to balance.

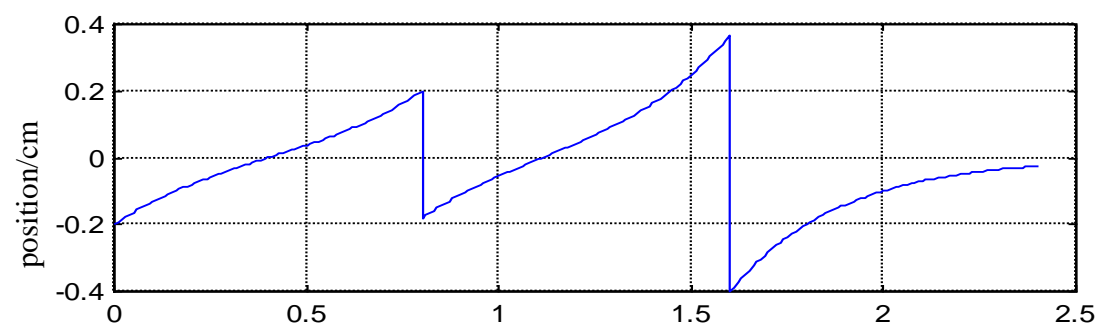

a) Centroid position

time (s)

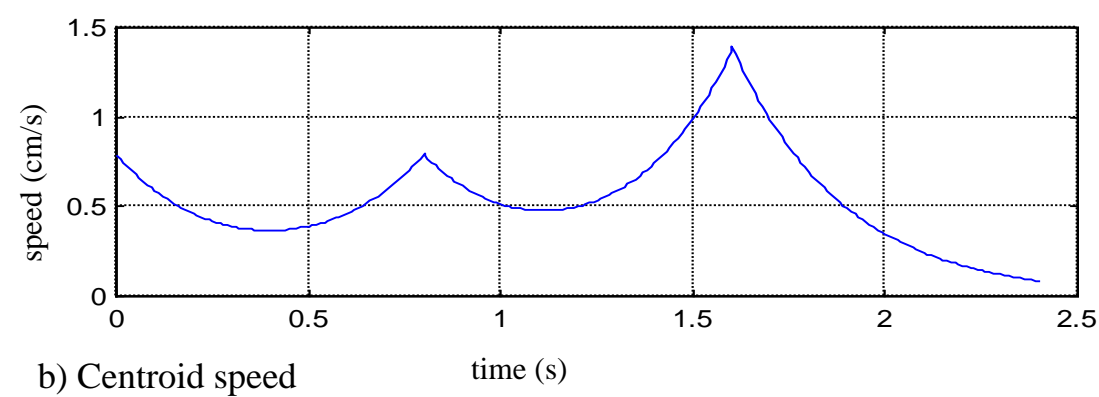

Figure 7: Total output response.

Table II: The states of centroid position and speed.

\begin{tabular}{|c|c|c|c|}
\hline$\rho$ & $\mathrm{C}$ & {$\left[x_{0}(i), \dot{x}_{0}(i)\right]$} & Running time \\
\hline$|\rho|<\rho_{s \tan d 1}$ & $\mathrm{C} 1$ & $-0.2,0.791$ & $0 \sim 0.8$ \\
\hline$\rho_{s \tan d 1} \leq|\rho|<\rho_{s \tan d 2}$ & $\mathrm{C} 2$ & $-0.18,0.791$ & $0.81 \sim 1.6$ \\
\hline$\rho_{s \tan d 2} \leq|\rho|<\rho_{\text {step }}$ & $\mathrm{C} 3$ & $-0.4,1.4$ & $1.61 \sim$ \\
\hline
\end{tabular}

\section{CONCLUSIONS}

Under normal circumstances, the control of biped robots is tracking control under weak interference. Strong interference causes falling down and losing balance.

In this study, strong and medium interference is considered. A multi-model stability control method for underactuated biped robots is proposed based on imbalance degrees. The main idea is to detect the position and speed of the centroid, and calculate the imbalance degrees. Corresponding control strategies are adopted according to the moving conditions of biped robots, in order to prevent falling down and restore posture and reference movement, eventually reaching a steady walking status.

\section{ACKNOWLEDGEMENTS}

This research was supported by Zhejiang Provincial Natural Science Foundation of China under Grant No. LY13F030004 and Science Technology Dept. of Zhejiang Province and Ningbo City under Grant No. 2014C50004.

\section{REFERENCES}

[1] Zhang, Z. (2010). Stable control strategy of the underactuated biped robot, $8^{\text {th }}$ World Congress on Intelligent Control and Automation, 6518-6523, doi:10.1109/WCICA.2010.5554197 
[2] Guan, Y. (2007). Humanoid Robots, Tsinghua University Press, Beijing

[3] Nguyen, K.-D.; Dankowicz, H. (2015). Adaptive control of underactuated robots with unmodeled dynamics, Robotics and Autonomous Systems, Vol. 64, 84-99, doi:10.1016/j.robot.2014.10.009

[4] Wieber, P.-B.; Chevallereau, C. (2006). Online adaptation of reference trajectories for the control of walking systems, Robotics and Autonomous Systems, Vol. 54, No. 7, 559-566, doi:10.1016/ j.robot.2006.04.007

[5] Chew, C.-M., Pratt, G. A. (2001). Adaptation to load variations of a planar biped: Height control using robust adaptive control, Robotics and Autonomous Systems, Vol. 35, No. 1, 1-22, doi:10.1016/S0921-8890(00)00130-5

[6] Suzuki, S.; Furuta, K.; Pan, Y.; Hatakeyama, S. (2001). Lazy VS-control strategy for passive walking, Proceedings of the $40^{\text {th }}$ IEEE Conference on Decision and Control, Vol. 2, 1392-1397, doi:10.1109/.2001.981085

[7] Collins, S.; Ruina, A.; Tedrake, R.; Wisse, M. (2005). Efficient bipedal robots based on passive-dynamic walkers, Science, Vol. 307, No. 5712, 1082-1085, doi:10.1126/science.1107799

[8] Geng, T.; Porr, B.; Worgotter, F. (2006). Fast biped walking with a sensor-driven neuronal controller and real-time online learning, The International Journal of Robotics Research, Vol. 25, No. 3, 243-259, doi:10.1177/0278364906063822

[9] Iida, F.; Minekawa, Y.; Rummel, J.; Seyfarth, A. (2009).Toward a human-like biped robot with compliant legs, Robotics and Autonomous Systems, Vol. 57, No. 2, 139-144, doi:10.1016/j.robot. 2007.12.001

[10] Ono, K.; Takahashi, R.; Shimada, T. (2001). Self-excited walking of a biped mechanism, The International Journal of Robotics Research, Vol. 20, No. 12, 953-966, doi:10.1177/ 02783640122068218

[11] Zhang, Z. (2011). Motion control of underactuated biped robots, Chinese patent, No. ZL 20091 0153337.5 (http://epub.sipo.gov.cn/patentoutline.action)

[12] Muscato, G.; Spampinato, G. (2007). Kinematical model and control architecture for a human inspired five DOF robotic leg, Mechatronics, Vol. 17, No. 1, 45-63, doi:10.1016/j.mechatronics. 2006.05.003

[13] Droniou, A.; Ivaldi, S.; Sigaud, O. (2015). Deep unsupervised network for multimodal perception, representation and classification, Robotics and Autonomous Systems, Vol. 71, 83-98, doi:10.1016/j.robot.2014.11.005

[14] Zhang, Z. (2012). The lateral motion control of underactuated biped robots, Chinese patent, No. ZL 20111 00496836, (http://epub.sipo.gov.cn/ patentoutline.action)

[15] Sheng, T.; Wang, J.; Ma, H.-X. (2008). Vertical posture control of point-connecting biped robots, Control Engineering of China, Vol. 15, No. 6, 703-706, 723, doi:10.3969/j.issn.16717848.2008.06.023

[16] Huang, Q.; Yokoi,K.; Kajita, S.; Kaneko, K.; Arai, H.; Koyachi, N.; Tanie, K. (2001). Planning walking patterns for a biped robot, IEEE Transactions on Robotics and Automation, Vol. 17, No. 3, 280-289, doi: $10.1109 / 70.938385$

[17] Yang, J.; Huang, Q.; Li, J.; Li, C.; Li, K. (2006). Walking pattern generation for humanoid robot considering upper body motion, Proceedings of 2006 IEEE/RSJ International Conference on Intelligent Robots and Systems, 4441-4446, doi:10.1109/IROS.2006.282078

[18] Canudas-de-Wit, C. (2004). On the concept of virtual constraints as a tool for walking robot control and balancing, Annual Reviews in Control, Vol. 28, No. 2, 157-166, doi:10.1016/ j.arcontrol. 2004.03.002

[19] Kajita, S.; Kanehiro, F.; Kaneko, K.; Yokoi, K.; Hirukawa, H. (2001). The 3D linear inverted pendulum mode: a simple modeling for a biped walking pattern generation, Proceedings of 2001 IEEE/RSJ International Conference on Intelligent Robots and Systems, Vol. 1, 239-246, doi:10.1109/IROS.2001.973365 